[Reflexiones y comentarios]

\title{
La epistemología de Quine: un análisis de la reificación
}

Quine's Epistemology: An analysis of reification

\author{
MARÍA DE LA ESPERANZA AGUILAR DE LA MORENA \\ Universidad de Málaga (España)
}

recibido: 10.10 .2018

aceptado: 04.06.2018

\section{RESUMEN}

El objetivo de esta investigación es el de realizar un análisis de la epistemología de Quine. Para ello, comenzaré planteando la problemática filosófica del empirismo que nuestro autor critica en su obra. Continuaré realizando un análisis de la teoría del conocimiento de Quine desde su tesis pragmatista, con la que explicaré cómo se da el conocimiento según sus conceptos fundamentales: la reificación y el holismo. La tesis que defenderé es que los problemas que han caracterizado a la metafísica y, en consecuencia, al empirismo son producidos por un mal uso de la reificación.

\section{PALABRAS CLAVE \\ REIFICACIÓN; PRAGMATISMO; HOLISMO; EPISTEMOLOGÍA}

\section{ABSTRACT}

The goal of this research is to analyze Willard V. O. Quine's epistemology. To that end I will begin by stating the philosophical problematic of empiricism that our author criticized in his work. I will continue it making an analysis of Quine's Theory of Knowledge from his pragmatist theses, which I will use to explain how knowledge occurs according with its fundamental concepts: reification and holism. The theses I will defend for is that the problems that have characterized metaphysics and, as a consequence, empiricism are produced by a bad use of reification.

KEYWORDS

REIFICATION; PRAGMATISM; HOLISM; EPISTEMOLOGY 


\section{INTRODUCCIÓN}

EN EL PRESENTE TRABAJO me dispongo a realizar un análisis de la epistemología de Willard Van Orman Quine. Para ello, comenzaré con una breve exposición que sirve de punto de partida. A partir de la mordaz crítica que Quine propone en «Dos dogmas del empirismo» (1950), se propone lo que él comienza por llamar un empirismo sin dogmas. Como sabemos, este empirismo posteriormente es catalogado como un pragmatismo, un giro que también he intentado recoger en mi investigación. Todo este proyecto nos obliga a recoger textos diversos de la filosofía de Quine, entre los que se encuentran algunos de importante peso filosófico, como «Dos dogmas del empirismo» (1950) o «El naturalismo, o el vivir por los propios medios» (1994), que serán centrales para este trabajo. Sin embargo, también recojo las nociones de enunciado observacional y categórico observacional, nociones básicas de su filosofía, las cuales aparecen en «Tres indeterminaciones» (1990).

Aunque las nociones de enunciado observacional y categórico observacional son fundamentales para sus investigaciones sobre el lenguaje, haré uso de sus tesis lingüísticas solo en la medida en la que sea necesario para la tesis epistemológica que he desarrollado. Por ello, las nociones centrales que he tratado son el holismo y la reificación. Si bien tradicionalmente se ha propuesto un esquema en el que el holismo era la clave de bóveda del pragmatismo de Quine, en mi argumentación he tratado de invertir el esquema profundizando en la reificación. De este modo el holismo pasa a ser la respuesta que Quine da para evitar los malos usos que se han dado en determinadas corrientes en la historia de la filosofía.

Esto nos conduce a la tesis final del trabajo, en la que contrastaré el problema metafísico con el problema del empirismo que, si bien parecen dos corrientes diametralmente opuestas, trataré de demostrar que su modo de proceder parte del mismo error. Por lo tanto, la conclusión a la que he llegado es que el problema que une a estas dos filosofías es el problema causado por una mala interpretación de la reificación.

\section{CRÍTICA A LOS DOS DOGMAS DEL EMPIRISMO}

El empirismo lógico se caracteriza por la conocida tesis que afirma que un enunciado es cognitivamente significativo sólo si posee un método de 
verificación empírica o es analítico ${ }^{1}$. Sin embargo, en «Dos dogmas del empirismo», Quine destruye estos dos principios a través de un análisis en el que afirma que ambos están sostenidos por tesis metafísicas.

El primer dogma que destruye es el de la distinción clásica entre analítico y sintético, que la filosofía arrastra y que es una distinción fundamental en la filosofía moderna de la que se hacen eco autores de peso tales como Leibniz o Kant. Los juicios analíticos son aquellos cuya verdad no depende de los hechos, sino de sus significaciones. Quine analiza las diferentes definiciones que le damos a los enunciados analíticos, un proceso que le lleva a cuestionar en primer lugar la definición; en segundo lugar, la intercambiabilidad; y, en tercer lugar, las reglas semánticas. Mediante este análisis llega siempre a la conclusión de que toda definición que demos de lo analítico siempre nos conduce a la misma afirmación: para todo juicio analítico, siempre hay un postulado previo que se contrasta observacionalmente. En palabras de Quine: «La verdad en sentido general depende a la vez del lenguaje y del hecho extralingüístico» (Quine 1962, 70). En las propias definiciones que damos de lo analítico encontramos debilidades que nos llevan a afirmar que la observación está presupuesta en ellas. Esta crítica a la distinción entre analítico-sintético la continúa Putnam en El desplome de la dicotomía hecho-valor y otros ensayos (2004). Sin embargo, Putnam sí que llegará a admitir que existen los enunciados analíticos, sólo que la distinción no es una cuestión de blanco o negro, sino de una escala gradual en la que a veces la respuesta es vaga: «Una distinción no es una dicotomía» (Putnam 2004, 23).

El segundo dogma del empirismo que Quine identifica se sigue de este primer dogma. Se trata del reduccionismo y llega a él a través del método de la verificación que nombré antes. El reduccionismo es la tesis que afirma que todo enunciado puede ser reducido a su contenido empírico, es decir, a su referencia a la realidad que descubrimos mediante la experiencia. Sin embargo, esta reducción nos hace caer en errores debido a la creencia ingenua de que existe una relación unívoca entre el enunciado y el objeto. Rudolf Carnap es uno de los primeros que intentó realizar amplios pasos hacia este principio dentro de su teoría lógica (Quine 1969,

1 «Analicity was thus to be a clear and precise idea, acceptable even to the most scrupulous of empiricist, which would play something of the philosophical role of the metaphysical ideas» (Hylton 2007, 48). 
74). Sin embargo, incluso los enunciados que eran más elementales sobre el mundo físico se topaban con el problema de que no podían superar un nivel esquemático (Quine 1962,73). Aunque esta creencia, después de los problemas con los que se enfrentó Carnap, haya sido destruida en la mayoría de pensadores, aún existe la creencia de que todo enunciado, siendo considerado de manera aislada a sus compañeros, puede tener confirmación o invalidación (Quine 1962, 75).

Alfred J. Ayer, siguiendo las tesis de Ludwig Wittgenstein, se propone dar un paso más en el conocimiento empírico. No sólo analiza la relación unívoca entre el enunciado y el objeto, sino que su análisis se centra en las relaciones lógicas tan presentes en el Tractatus logico-philosophicus (1921). Se enfrenta a las mismas dificultades que Carnap, aunque empleando la lógica para intentar solventarlas. Su uso de la lógica viene dado por lo que Ayer denomina la teoría de la coherencia de la verdad que «no se ocupa de la definición de la verdad y la falsedad, sino sólo de los medios mediante los cuales estas se determinan» (Ayer 1965, 236). Sin embargo, pese a los esfuerzos que realiza sus resultados vuelven a enfrentarse a los mismos problemas que Carnap.

Como vemos, el empirismo no está exento de problemas. Estos dos dogmas señalados son, según Quine, prejuicios basados en tesis metafísicas que no deberían estar presentes en una teoría del conocimiento. Nos encontramos, pues, con una problemática que es arrastrada desde el comienzo de la filosofía y que Quine demuestra que sigue presente incluso en la corriente que intenta destruirla. El empirismo no está exento de prejuicios metafísicos, más bien «la convicción de que esa línea debe ser trazada es un dogma nada empírico de los empiristas, un metafísico artículo de fe» (Quine 1962, 70).

Mi propósito ahora es recuperar la respuesta que da Quine como alternativa a estos fallos o, como él lo llama, un empirismo sin dogmas. Para ello, el método elegido es el de rastrear las tesis pragmatistas de Quine a lo largo de sus obras que considero de mayor relevancia para el tema que nos ocupa. Intentaré sistematizar su respuesta rastreando sus tesis principales. Con este objetivo, Quine destruye en primer lugar el empirismo, analizando en profundidad sus problemas centrales, para plantear un nuevo modo de teoría del conocimiento habiéndolos superado. De este modo, se podrá comprobar la fuerza que tiene la reificación dentro de la propuesta de 
Quine, como un nuevo inicio de un empirismo mucho más cauteloso gracias a la tesis del holismo. Esta vez, liberado no solamente de sus dogmas, sino de aquello que los provoca: una mala interpretación de la reificación fruto de la incapacidad de tomar distancia de ella para poder interpretarla de manera correcta.

\section{Pragmatismo}

El pragmatismo en la obra filosófica de Quine supone un eje central para entender sus tesis sobre epistemología. En este primer apartado intentaré realizar un rastreo de su tesis pragmatista que considero de mayor relevancia por diferentes textos centrales de su obra. El objetivo de esto es el de desarrollar la tesis de Quine acerca de la ciencia para poder acceder a una definición de conocimiento. Para ello, comienzo con una primera aproximación a su pragmatismo, base que sirve para explicar posteriormente el modo en que creamos el conocimiento mediante enunciados observacionales. Esta definición nos lleva a lo que Quine denomina los categóricos observacionales, sin los cuales no podríamos construir hipótesis contrastables. Todo ello siempre a la luz del naturalismo que Quine desarrolla al final de su vida. Esto nos permite contrastar las tesis del naturalismo de Quine con las tesis empiristas y sus dos mayores falacias: la distinción analítico-sintético y el reduccionismo.

\section{III.1. El PRAGMATISMO DESDE «DOS DOGMAS DEL EMPIRISMO»} A «El Naturalismo, O EL VIVIR POR LOS PROPIOS MEDIOS»

El pragmatismo de Quine puede rastrearse comenzando por «Dos dogmas del empirismo». Es aquí donde nuestro autor muestra por primera vez su inclinación pragmática, que continuará y se afianzará con el tiempo. Quine nos ofrece su visión de lo que sería un nuevo empirismo liberado de sus dos viejos dogmas que hemos tratado anteriormente y es a partir de la crítica desde donde se percibe su talante pragmatista. Al final del artículo, como ya señalé, Quine defiende que nuestro conocimiento del mundo, incluida la ciencia, no es más que un edificio construido por nosotros mismos, mediante el cual intentamos sistematizar nuestra concepción de la realidad. «La totalidad de lo que llamamos nuestro conocimiento [...] es una fábrica construida por el hombre y que no está en contacto con la experiencia más que a lo largo de sus lados» (Quine 1962, 77). 
Un punto de partida para entender las diferencias del pragmatismo con el empirismo es explicar que las experiencias no se encuentran de ninguna manera ligadas a un enunciado. De este modo, emerge un nuevo problema: el de la referencia de los enunciados. Para el pragmatismo, «debemos concluir que los objetos, del tipo que sean, figuran sólo como nodos neutrales en la estructura [...]. Podemos cambiar arbitrariamente los valores de nuestras variables, lo que nuestros nombres designan y los que nuestros predicados denotan sin perturbar la evidencia» (Quine 2001, 138). Según él, los enunciados no están ligados unívocamente con los objetos a los que se refieren, sino que adquieren su sentido a la luz de otros enunciados. Eso que parece un problema irresoluble dentro de cualquier postura filosófica, para Quine no lo es, ya que este problema atañe directamente a la metafísica, pero no así al pragmatismo. Debido a esto, nos propone una nueva forma de entender esta referencia. Es por ello que, a esta teoría de Quine, se la considera un pragmatismo holístico. De este modo, todos los enunciados están ligados unos con otros, formando un plexo de relaciones dentro del cual no se puede entender ninguno de ellos individualmente (Quine 2001, 91).

En este plexo de relaciones, no todos los enunciados poseen la misma validez epistémica. El canon que propone Quine para saber qué enunciados tienen mayor importancia epistémica es juzgándolos a la luz de su capacidad explicativa, aunque posteriormente lo reformulará y reducirá la exigencia a meramente predictiva (Quine 2001, 129-130). Este holismo que he mencionado con anterioridad cala tan hondo en la filosofía de Quine que incluso el valor epistémico de los enunciados depende de su relación con otros enunciados. Estas relaciones hacen que el conjunto de los enunciados de cada individuo se organice de manera jerárquica. La jerarquización llevada al máximo es lo que hace que Quine convierta esta teoría en un sistema. Ya que la referencia ha dejado de ser un medio útil para proporcionar un conocimiento fiable, Quine sostiene que, si un enunciado es superior a otro, es porque ha demostrado ser más eficaz en nuestro proyecto de elaborar un sistema del mundo: «Pero en cuanto a fundamento epistemológico, los objetos físicos y los dioses difieren sólo en grado, no en esencia» (Quine 1951, 79). Con esta sentencia, Quine hace especial hincapié en que los objetos de las tesis físicas son epistemológicamente superiores por el simple hecho de que favorecen en mayor 
grado nuestro manejo de la experiencia sensible a través de su capacidad explicativa. Es decir, «porque ha probado ser más eficaz que ellos como procedimiento para elaborar una estructura manejable en el flujo de la experiencia» (Quine 1962, 79).

No convendría terminar esta explicación sin avanzar superficialmente en el análisis del holismo que es considerado por sus coetáneos la característica principal de este pragmatismo. Como he señalado anteriormente, los enunciados observacionales adquieren su sentido por la relación que tienen unos con otros. Este holismo hace que su relación sea muy peculiar. En el momento en el que un enunciado quede refutado, como el resto de los enunciados con el que mantiene relación entran en juego, el modo más sencillo de resolver esta situación es comprobar de nuevo este enunciado observacional. En caso de revisión, se ha de tratar siempre que mantenga una coherencia explicativa con el resto de los enunciados siguiendo la máxima de mutilación mínima ${ }^{2}$. Esto se hace con la pretensión de que la estructura con la que el individuo maneja el flujo de la experiencia no sufra un daño irreversible. Por esto, esta tesis es de talante pragmatista, puesto que lo más importante siempre es mantener la estructura. Tampoco puede pasar desapercibido que el hecho de poder contrastar el enunciado observacional con otros significa que no posee, como he señalado antes, una relación directa con el mundo, por lo que, en un sentido pleno de la palabra, no existe tesis ontológica alguna en la filosofía de Quine.

En el subapartado siguiente analizo las condiciones necesarias para la producción de enunciados observacionales: la reificación y la intersubjetividad. Esto nos permitirá pasar a los tres conceptos tan particulares que utiliza Quine: los enunciados observacionales, los categóricos observacionales y el holismo. No obstante, la reificación es parte fundamental de este proyecto, pues considero que al ser primigenia es una de las piedras angulares de la propuesta de Quine.

2 «Debemos también seguir la pista de conjuntos de enunciados en otra parte, en nuestra teoría global, que impliquen a esos enunciados recientemente revocados, pues ellos deben ser desactivados también. Continuamos así hasta que la consistencia parezca restaurarse: tal es la mutilación que el máximo de mutilación mínima intenta minimizar» (Quine 2001, 92). 


\section{III.2. REIFICACIÓN E INTERSUBJETIVIDAD}

Paso ahora a realizar el análisis de los enunciados observacionales que mencioné antes. Para ello, es preciso comenzar puntualizando que en la visión naturalista de Quine, los enunciados observacionales son la forma más fiable de conocimiento, precisamente porque «los enunciados observacionales tienen sus antecedentes en el canto de los pájaros y en el grito de los monos» (Quine 2001, 131). Como mencionamos con anterioridad, los enunciados no están en relación directa con los cuerpos sobre los que versan, sino que existe una red de relaciones que otorga sentido a cada enunciado. Quine completa un poco más esta información añadiendo que los enunciados, además de estar relacionados unos con los otros, son lo que están en relación directa con las entradas neuronales. A partir de estas entradas neuronales es donde comienza de manera segura nuestro conocimiento. Es decir, dentro de todo nuestro conjunto de conocimiento, las entradas neuronales serían lo que funciona de filtro entre el mundo y nuestros enunciados del mismo. Por ello, la carga ontológica en Quine se desdibuja, porque no se trata de enunciados que versan sobre los cuerpos del mundo, sino que «esta venerable teoría de la persistencia y recurrencia de los cuerpos es característica del uso de la reificación al integrar nuestro sistema del mundo» (Quine 2001, 85). Se trata, en cambio, de los objetos de la realidad, «¿Debemos por tanto concluir que la verdadera realidad rebasa nuestra comprensión? No, eso sería renunciar al naturalismo. Se trata, más bien, de que la noción de realidad es en sí misma parte del aparato» (Quine 2001, 141). Es decir, que los enunciados hacen referencia a los objetos que están inmersos en ese plexo de relaciones que llamamos realidad. ${ }^{3}$ Pero no hemos de pensar que este sentido es arbitrario, sino que está fuertemente condicionado por nuestras capacidades cognitivas.

¿Cómo es posible, entonces, que hablemos de un conocimiento contrastable? Dentro de la tesis naturalista de Quine, existen dos mecanismos o procesos mediante los cuales se puede dar el conocimiento. Estos son el paso siguiente de explicación, después de haber precisado la relación de los enunciados observacionales con las entradas neuronales. En primer lugar, vemos que dentro de nuestro aparato cognitivo poseemos la inclina-

3 «Epistemology in this sense is concerned not only with how we can know anything about the world, but also with the question how we can say or believe anything -true or false- about the world at all» (Hylton 2007, 82). 
ción innata a postular objetos, sobre todo aquellos que poseen porciones corpóreas del campo visual. (Quine 2001,132). Esta inclinación, conocida como reificación, se hace a partir de lo que Quine llama los cuerpos del mundo, los cuales no se pueden conocer directamente. Esto hace que considere inútiles las discusiones puramente ontológicas, puesto que su única vía es a partir del uso de las variables ligadas (Quine 1962,39). Su carácter naturalista llega hasta tal punto que incluso esta capacidad o inclinación la sustenta a nivel genético, siendo nuestros genes los que nos permiten tener una visión parecida del mundo (Quine 2001, 131). Esta reificación es el paso previo que damos antes de poder componer los enunciados observacionales. Es esencial porque, al no tener los propios enunciados por sí mismos una relación con los cuerpos sobre los que versan, esta capacidad es la que nos posibilita crearlos, dando inicio a la red de relaciones posterior.

Para Quine, este punto es tan relevante que incluso llega a afirmar que la realidad forma parte de este proceso de reificación. Cuando decimos que nuestros enunciados se refieren a objetos de la realidad, nos referimos a esos cuerpos que han pasado a formar parte de la estructura de relaciones con las que manejamos el flujo de la experiencia. Quine, dentro de esta tesis sobre la fundamentación de los enunciados observacionales, asevera que la realidad es un concepto más, reificado por nuestro aparato conceptual a través de ciertas entradas neuronales (Quine 2001, 135). Por lo tanto, lo único que tiene de particular la realidad respecto a las demás reificaciones es que ésta supone el telón de fondo donde situamos a las demás. En esta medida, rompe con la tradición filosófica imperante que trata al individuo como algo sobrepasado por cierta realidad preexistente que lo supera. Para Quine, ésta no podría trascendernos en la medida en la que ella misma es postulada por nosotros mismos, y es aquí desde donde considero que parten las críticas de Quine al empirismo y sus posteriores tesis pragmáticas.

Es necesario, sin embargo, puntualizar que no se trata de algo sucesivo, sino simultáneo. No sería correcto decir que en primer lugar se construyen los objetos y luego los reificamos. En efecto, reificar es una condición necesaria de nuestro conocimiento, y por eso siempre está presente. El papel de los enunciados en el desarrollo del conocimiento es que nos permiten parcializar esa reificación en objetos individuales, lo cual nos permite afinar nuestra capacidad predictiva y explicativa. La forma que 
existe entonces para que seamos capaces de comenzar a sistematizarlos es mediante la reificación y mediante el aprendizaje: el primero en tanto que es condición natural; el segundo, dado que nacemos inmersos dentro de una comunidad lingüística (Quine 1968, 94). Al niño, pues, se le presenta la realidad como un todo uniforme, incapaz de diferenciar los distintos objetos. Es esencial para Quine, llegados a este punto, señalar la importancia que posee la intersubjetividad en este proceso, ya que la realidad que se presenta como un todo para el niño, es posible sistematizarla y comenzar a jerarquizarla a través de los enunciados que este aprende de sus mayores ${ }^{4}$. Se trata de un proceso en el que, mediante ese aprendizaje de enunciados, el niño es capaz de adquirir el lenguaje cognoscitivo. Por ello es esencial, tanto la posibilidad de reificación, como la intersubjetividad que posibilita el conocimiento que tradicionalmente ha sido confundido con la objetividad. Esta, considero, es otra de las tesis fundamentales por las que Quine se diferencia de la tradición empirista. Debe haber un veredicto por parte de todos los hablantes que comparten el mismo uso de la lengua sobre los enunciados que lance un individuo.

Una vez que se han explicado las dos condiciones que se deben dar para que los enunciados observacionales sean posibles, volvamos a la característica de la que partía anteriormente: los enunciados observacionales no tienen una relación directa con los cuerpos, sino que se trata de una relación con las entradas neuronales, que son las que construyen los objetos. Esta es la base de la que parten los enunciados observacionales: provienen de las entradas neuronales en combinación con la reificación de objetos y con la intersubjetividad que los caracteriza.

\section{III.3. De LOS ENUNCIADOS OBSERVACIONALES A SU CONTRASTACIÓN}

Este análisis de cómo se posibilita el conocimiento nos clarifica algo más la desconexión que existe entre nuestros enunciados y el mundo. Al haber visto que el concepto de realidad es una consecuencia de cómo se da el conocimiento en cada individuo, sabemos que esta desconexión se debe precisamente a que no hay un conocimiento certero de un mundo

4 «El aprendizaje contextual de esas varias partículas procede simultáneamente, o así lo podemos suponer, de tal modo que todas van ajustándose gradualmente unas a otras, hasta producirse un esquema coherente de uso que concuerda con el de la sociedad» (Quine 1968, 106). 
ontológico simétrico a la realidad. En su lugar, recibimos ciertos estímulos mediante las entradas neuronales, los cuales son convertidos en enunciados observacionales a través de la reificación y la intersubjetividad. Los enunciados observacionales «constituyen nuestra introducción al lenguaje, pues son las expresiones que podemos aprender a usar directamente, sin hacerlo de los demás. A través de ellas es como el lenguaje y la ciencia absorben su contenido empírico» (Quine 2001, 37). Es por esto que, una vez aclarado cómo surgen, ahora podemos seguir analizando los enunciados observacionales para ver cómo se relacionan unos con otros y dan lugar al conocimiento. Es importante señalar que lo que busca Quine es intentar dar con el mejor modo de contrastación, para que podamos hablar del conocimiento del modo más fiable posible. No olvidemos que la pretensión de este apartado es la de explicar la importancia que tiene la reificación de Quine dentro de su epistemología. Para ello, debo volver al aprendizaje de los enunciados observacionales.

Como dije anteriormente, el aprendizaje es el medio por el cual el niño se inserta en el lenguaje cognoscitivo, ya que le posibilita sistematizar los enunciados observacionales. Sin embargo, la intersubjetividad de la que depende el aprendizaje no es sólo importante por esto. El compartir un mismo vocabulario es lo que crea el canal mediante el cual se posibilita la comprobación de hipótesis científicas (Quine 2001, 133). Es necesario saber entonces qué es para Quine una hipótesis científica y cómo surgen, y para ello es necesario introducir el concepto de categórico observacional. Los categóricos observacionales son las hipótesis primordiales y su característica principal es que se componen de enunciados observacionales. Aunque en su texto «El naturalismo, o el vivir por los propios medios» no especifica mucho más allá de esta definición, en «Tres indeterminaciones» sí que realiza una explicación más profunda de los categóricos observacionales. Al tratarse de pares de enunciados observacionales, se trata de generalizaciones que se componen de observables y que, además, siempre aparecen en forma de condicionales (Quine 2001, 87). Un ejemplo de un categórico observacional sería: «Cuando nieva, hace frío». Una característica esencial para que se dé la comprobación y contrastación de los categóricos observacionales es, por tanto, que sean reducibles sus componentes a enunciados observacionales. Esta reductibilidad es lo que posibilitará la comprobación o refutación del categórico en cuestión. De 
este modo, Quine salva la crítica de la verificabilidad y el reduccionismo que supone una falacia del conocimiento empírico como tal. Esto se debe a que, para Quine, lo único que puede ser sometido a comprobación son los enunciados observacionales que, como ya he dicho, son la forma más fiable de conocimiento, al ser lo que está más íntimamente relacionado con la entrada neuronal.

A través de los categóricos observacionales es como se da comienzo a la red de relaciones de enunciados observacionales de la que hablé anteriormente. Es la manera en la que puede darse el conocimiento más allá de los enunciados observacionales ${ }^{5}$. Una vez señalado a grandes rasgos qué son y para qué sirven, es necesario señalar la diferencia entre categóricos observacionales primitivos y categóricos observacionales objetuales. Por un lado, los categóricos observacionales primitivos son condicionales de los que surgen generalizaciones irreductibles. Son los que el niño será capaz de aprender al poco tiempo de sus primeros enunciados observacionales, ya que no incluyen cuantificación ni variables ligadas y un ejemplo sería el anterior de la nieve. Se trata de un condicional en el cual los dos componentes que lo constituyen no están ligados más que por la unión que el propio categórico observacional implica. Son, por lo tanto, poco agresivos contra la expectativa generalizada de un grupo de hablantes de la misma lengua. «Los categóricos observacionales primitivos, a diferencia de los objetuales, son expresiones ontológicamente inocentes respecto a la expectativa generalizada» (Quine 2001, 88). Por otro lado, los categóricos observacionales objetuales dependen de la cuantificación o, al menos, de algo que equivalga a ello. Son relaciones más sofisticadas en las que hay algún tipo de relación entre la segunda parte del condicional y la primera. Un ejemplo que usa el propio Quine en «Tres indeterminaciones» de este segundo tipo sería: «Cuando un sauce crece al borde del agua, se inclina sobre ella» (Quine 2001, 88). En este categórico observacional podemos comprobar cómo el segundo componente se refiere al sauce que ya aparece en el primer componente. La relación es, por lo tanto, mucho más comprometida, ya que el objeto del segundo enunciado no se puede entender

5 «Generaciones de lingüistas han subrayado con razón que el rasgo distintivo del lenguaje, rasgo no compartido por los sistemas de señalización de los animales inferiores, es su productividad de combinaciones. Aprendemos modos de composición como aprendemos palabras, y así somos capaces de producir expresiones complejas nunca oídas con anterioridad, o de responder a ellas» (Quine 1998, 76). 
sin el objeto del primer enunciado.

Una vez señalada la diferencia entre los dos tipos de categóricos observacionales, estamos en condiciones de dar un paso más hacia la comprobación de dichos categóricos, que es lo que nos ocupa. Para llegar a ello hay que reparar en una característica peculiar que poseen los categóricos observacionales. En el anterior ejemplo del sauce, la manera en la que llegamos a sugerir el categórico observacional es a través de una observación casual: el percibir sauces inclinados sobre un arroyo. El categórico observacional nos inducirá a una hipótesis teórica que tendremos que inventar, en este caso sería: «La raíz de un sauce alimenta principalmente su propio lado del árbol». Ambas cosas tendrán que ir a su vez ligadas a ciertas teorías previas, pero lo esencial es que hemos creado un categórico observacional a través de la observación y enunciados observacionales (Quine 2001, 88). La característica peculiar de la que hablaba es que el camino se puede recorrer a la inversa. Es decir, los categóricos observacionales nos pueden servir a su vez no sólo para ser comprobados y avanzar en el conocimiento, sino para deducir ciertos enunciados observacionales. El proceso es complejo y por ello era necesaria la distinción entre categóricos observacionales primitivos y objetuales (Quine 2001, 132).

Un categórico observacional primitivo puede ayudarnos a simular la referencia objetiva hasta el punto en el que podamos afirmar el enunciado observacional al que va ligado y, posteriormente, otros categóricos observacionales más sofisticados. Esto se debe a que con un categórico que sea primitivo y tan sólo afirme, por ejemplo, «Siempre que bípedo implume, mamífero», el niño podrá dar comienzo a la reificación como consecuencia de subsumir el primer conjunto de escenas bajo el segundo; en nuestro ejemplo se subsumiría a todos los bípedos implumes en los mamíferos. Esto llevaría así a los enunciados observacionales reificados del categórico primitivo, que podrán posteriormente dar lugar a un categórico objetual más sofisticado como «todos los bípedos implumes son mamíferos». Ahora puede verse con más claridad la utilidad de la reificación en el proceso del conocimiento, ya que posibilita agrupar distintas cualidades hasta el punto de poder afirmar que esto y aquello constituyen la misma porción de la escena.

Como vemos todo este proceso de los enunciados y categóricos observacionales en su relación holística no es más que un proceso para evitar 
la reificación en exceso, tanto de los enunciados, como de los conceptos. Así, los enunciados deben tener esta forma tan peculiar que acabamos de analizar porque lo que se busca es maximizar su capacidad de revisión. Esta capacidad de revisión presente en todo el esquema de Quine se debe precisamente a los problemas de la reificación en la historia de la filosofía.

\section{III.4. REFUTACIÓN Y HOLISMO A LA LUZ DEL CONTENIDO EMPíRICO}

Una vez establecido lo que son los categóricos observacionales y para qué sirven, podemos pasar ahora a analizar cómo se produce el proceso de prueba y contrastación de los mismos. Ya que los categóricos observacionales tienen la forma de condicionales, se prueban experimental y observacionalmente si, al darse el segundo componente, se da también el primero. Si es así se mantiene por el momento y si no, queda refutado. Mediante este proceso es como se prueban a su vez hipótesis más sofisticadas creadas a partir de categóricos observacionales, como las hipótesis científicas.

Este proceso consistiría en establecer una relación entre la hipótesis y una serie de enunciados ya aceptados y deducir de tal unión un categórico observacional comprobable que no se pudiera obtener con otros medios. Así es como Quine afirma que es el procedimiento del método hipotético-deductivo: «Aun así, la deducción y comprobación de categóricos observacionales es seguramente la esencia del método experimental, del método hipotético deductivo; el método, en palabras de Popper, de las conjeturas y las refutaciones» (Quine 2001, 134). Esto no tiene que inducirnos al error de considerar que las hipótesis científicas tienen que basarse solo en la predicción. Quine propone la comprobación como la guía para establecer las teorías científicas, sin embargo, abre la puerta a establecer como válida cualquier hipótesis que, aunque por sí misma no sea siquiera comprobable, unida a otras anteriores produzca una hipótesis que sí lo sea. Quine llega a afirmar que, de hecho, la mayoría de la ciencia utiliza en gran medida estas hipótesis (Quine 2001, 135).

Vemos pues que las hipótesis son contrastables mediante los categóricos observacionales que producen y, por lo tanto, no hay verificación concluyente, sino tan sólo refutación. Por ello el método hipotético-deductivo es el correcto para Quine. Llegados a este punto, es crucial recuperar el holismo que expliqué al comienzo del apartado. Este holismo en el que 
todos los enunciados observacionales y, por ende, los categóricos observacionales están interconectados y dependen unos de otros es lo que hace a Quine postular el máximo de mutilación mínima. Mediante esta máxima, tan sólo pondremos en cuestión el enunciado que parezca sospechoso de ser refutado. Si cuando se refute la teoría en cuestión continúa siendo sospechosa, se atenderá al siguiente enunciado, y así hasta que sea irrefutable. Lo que guía el método de comprobación es, por tanto, el no desequilibrar demasiado el esquema cognitivo, lo cual nos hace determinar el fuerte modo en el que el pragmatismo está presente en todo el proceso.

La máxima de mutilación mínima va acompañada a su vez de la máxima de simplicidad, según la cual es importante tener en cuenta la simplicidad de la teoría que resulte de las refutaciones realizadas (Quine 2001, 90). Estos dos principios, maximización de la simplicidad y minimización de la mutilación, son los dos principios que guían la ciencia y el progreso del conocimiento en busca de predicciones futuras. Vemos cómo Quine termina concluyendo que el contenido empírico de un enunciado, no se define entonces como una simetría con el mundo exterior, sino como «el conjunto de todos los categóricos observacionales sintéticos implicados, más todos los categóricos observacionales adicionales que pueden ser estimulativamente sinónimos con cualquiera de ellos» (Quine 2001, 94). De este modo, y rompiendo con los dogmas clásicos del empirismo, los términos dejan de parecernos vehículos de significado, de hecho, ni siquiera los enunciados mismos lo son. Ahora, en cambio, la carga la debemos situar en los conjuntos de esa red de relaciones, puesto que el contenido empírico de un enunciado o un conjunto de enunciados depende, únicamente, de su susceptibilidad de comprobación, alejada ahora de la verificabilidad y el reduccionismo, y abierto a un nuevo campo gracias al holismo y al uso prudente de la reificación.

\section{FILOSOFÍA Y REIFICACIÓN}

Gracias al análisis que he llevado a cabo en el apartado anterior, nos encontramos ahora en condiciones de analizar en profundidad el problema del empirismo que Quine critica en su obra. Se ha realizado una recuperación de las tesis más importantes de nuestro autor sobre su teoría del conocimiento y, a la luz de nuestros argumentos se puede concluir la importancia que posee el pragmatismo que aparece siempre como telón de 
fondo para su epistemología. El holismo ha supuesto el eje central en todas las interpretaciones de la filosofía de Quine, con el fin de escapar a los problemas del empirismo. No obstante, en este análisis hemos visto cómo la relevancia de este punto viene dada por la noción de reificación, convirtiéndose en el punto clave de su filosofía. Sin embargo, ahora se deben analizar los problemas del empirismo y de la tradición filosófica en general más a fondo. Para ello, es inevitable acudir al concepto de reificación que fue introducido al comienzo de la explicación del pragmatismo de Quine y que nos ha acompañado a lo largo del análisis. Mi propuesta parte de que es precisamente esta capacidad la que nos induce a los errores.

Para poder hablar de los problemas del empirismo y verlos a la luz de la crítica de Quine, es necesario primero hablar de la metafísica. La metafísica es criticada por Quine en diversos textos, principalmente en «Acerca de lo que hay». Esto se debe al fuerte rechazo que mantiene con cualquier compromiso ontológico como consecuencia de su concepción holista del conocimiento. Es necesario pararnos en la metafísica antes de continuar con el empirismo lógico que nos ocupa porque precisamente los problemas del empirismo son consecuencia de dos prejuicios metafísicos, a pesar de que sea lo que critica con tanta vehemencia el empirismo lógico. Después del análisis que realiza Quine de cómo se da el conocimiento a través de los enunciados observacionales, los categóricos observacionales y el holismo que caracteriza la relación entre todos ellos, es más sencillo apreciar por qué las tesis metafísicas caen en error.

Al comienzo del apartado anterior me detuve a explicar nuestra capacidad de reificación como una de las dos condiciones que se deben dar para que se produzcan los enunciados observacionales y, en definitiva, el conocimiento. La reificación apareció entonces como la capacidad y la inclinación innata de nuestra especie de reificar los cuerpos del mundo. Es decir, la capacidad de postular objetos, con preferencia por aquellos con porción corpórea dentro del campo visual. Dije entonces que lo que se reifica son los cuerpos del mundo y que estos se caracterizan por no poder ser conocidos directamente. Dejé claro también que esta capacidad la tenemos al igual que la poseen todos los mamíferos, por lo que la explicación que debe darse de la posesión de esta inclinación debe ser a nivel genético. La capacidad de reificar nos posibilita el hacer enunciados observacionales y hacer que los cuerpos del mundo pasen a ser objetos 
de la realidad reificada. Es necesario aquí advertir que Quine en ningún momento habla del mundo como lo que hay fuera de nuestro aparato conceptual. El mundo, los cuerpos, la realidad y los objetos son todos conceptos postulados necesarios para la jerarquización de nuestro esquema. A simple vista, podría parecer que, al formar la realidad y los objetos a partir del mundo y los cuerpos, estos últimos no son reificados, sino que se encuentran en el mundo externo. Sin embargo, los cuatro conceptos forman parte del conjunto de objetos que reificamos y que mantenemos por su capacidad explicativa y predictiva, manteniéndolos al margen del compromiso ontológico.

En «Acerca de lo que hay» Quine señala que el principal problema de las tesis metafísicas es la confusión que poseen entre nombres y significación ${ }^{6}$. No es lo mismo nombrar y significar y, por ello, el principal problema de la metafísica es precisamente creer que todo lo que puede ser nombrado puede ser, por ello, significado. Quine demuestra a través de la teoría de Bertrand Russell de las descripciones singulares, que «todo lo que puede decirse con la ayuda de nombres puede decirse también en un lenguaje que no los tenga» (Quine 1962, 39). De hecho, cuando decimos que algo es una entidad significa tan sólo que estamos afirmando eso como valor de una variable. Aquí refuerza Quine su tesis sobre la importancia de las variables ligadas en la significación y con esto, rompe con la confusión entre nombre y significación. Sin embargo, pienso que, si entendemos esto a la luz de la reificación, podemos realizar un análisis mucho más profundo de las tesis de Quine. La confusión de pensar que nombrar y significar son lo mismo es una consecuencia de la reificación. Más concretamente, de una mala interpretación de la reificación a la luz de las tesis de Quine. Esta capacidad en sí misma no es negativa, al contrario: posibilita el conocimiento. Sin embargo, una mala interpretación de la reificación puede conducir a engaños. El error en el que cae la metafísica es creer que por el simple hecho de poder reificar -algo que se logra a través de los nombresya se está significando. El hecho de ser capaces de postular los objetos y nombrarlos se presta a confusión y, de este modo, es precisamente donde se cree encontrar la significación de los objetos. Este error nos ha acompañado durante toda la historia de la filosofía, y el empirismo se presentó

6 «Quine emphasizes that being meaningful is to be distinguished from designating anything» (Parsons 2011, 4). 
en su momento como la solución a esto.

El empirismo lógico surge, entonces, como una mordaz crítica hacia el discurso de la metafísica y hacia todas las preguntas filosóficas que estaban contaminadas hasta entonces, según ellos, de tintes metafísicos. Para los empiristas, las proposiciones metafísicas eran pseudoproposiciones carentes de sentido. Como mencioné, para no caer en los mismos errores, el empirismo se basa en el reduccionismo y la existencia de enunciados analíticos para conseguir conocimiento fiable. Sin embargo, los reiterados intentos de Carnap por reducir al contenido empírico los enunciados se toparon con dificultades irresolubles. Estos errores pueden ser analizados del mismo modo a la luz de la reificación como causante de la confusión inicial. Me centraré en Carnap y Ayer como representantes de esta corriente filosófica y me valdré de sus tesis para trasladar el problema de la reificación.

Carnap, por un lado, mantiene que las representaciones de un objeto han sido confundidas, durante toda la historia del pensamiento, con las representaciones de un hecho. Para el alemán, el problema de las proposiciones ligadas a un lenguaje psíquico es que «con él se expresan las representaciones concomitantes de un objeto, que como tales no representan ningún hecho y por eso no pueden constituir el contenido de una proposición» (Carnap 1990, 35). De este modo, la metafísica lo que haría es precisamente expresar las representaciones de un objeto mediante pseudoproposiciones carentes de contenido. Así, para Carnap, el contenido empírico del mundo es en lo que se tiene que centrar la visión científica del mismo para poder avanzar. El contenido empírico del mundo es indiscutible en sus tesis. Por otro lado, Ayer, al ver que los intentos de Carnap estaban fracasando, se centra en la relación lógica ${ }^{7}$ de los enunciados para sustentar el contenido empírico: «Se simplificará nuestro cometido si podemos hacer una distinción entre las proposiciones empíricas, cuya verdad o falsedad sólo pueden determinarse averiguando la verdad o falsedad de otras proposiciones, y aquellas cuya verdad o falsedad puede determinarse directamente por observación» (Ayer 1965, 233). De aquí viene el reduccionismo característico de Ayer, ya que para poder establecer el significado de una

7 «On the other hand, he rejected the traditional philosophical study of scientific knowledge and mathematics in particular, because of its failure to reduce mathematics to pure logic» (Ervas 2012, 2). 
proposición es necesario transformarla hasta que tan sólo obtengamos palabras con significados ostensibles. Es decir, una reducción de todo el enunciado en su referencia a la observación. Sin embargo, aunque el planteamiento del problema es totalmente diferente, la causa de la equivocación del empirismo lógico es exactamente la misma que la de la metafísica.

La respuesta que da el empirismo a ese afán metafísico de sobrecargar ontológicamente a partir de los nombres se basa en negar todos esos conceptos y afirmar que el único modo de conocer es poner la mirada en el mundo empírico. Sin embargo, después de haber realizado las distinciones quineanas entre mundo y realidad, sabemos que no podemos hablar del mundo sin tener en cuenta que es un enunciado que forma parte del plexo de enunciados y categóricos observacionales. Nos topamos con el mismo problema que con la metafísica: el engaño de la reificación. En este caso no se les puede culpar a los empiristas de reificar los conceptos, de esto se alejan con total rotundidad. Sin embargo, no están escapando del auténtico problema, puesto que la respuesta que están dando no supone una alternativa real. En lugar de revisar cuál era la causa del problema metafísico, simplemente se centran en atacar sus consecuencias. Con la pretensión de evitar el uso de conceptos metafísicos que consideran innecesarios, acaban cayendo en el mismo error de reificación. En este caso, el empirismo lleva a cabo una reificación total de los cuerpos, convirtiéndolos en el único modo fiable de conocimiento.

Vemos cómo ha cambiado la referencia de la reificación, pero el método de fondo sigue siendo el mismo. Por ello Quine afirma que el empirismo no está libre de prejuicios metafísicos, dado que la auténtica crítica hacia la metafísica debería ir hacia el uso de la reificación y no hacia los problemas que surgen de la propia metafísica. Es aquí precisamente donde somos capaces de valorar la virtud de Quine: la crítica a la distinción entre analítico y sintético no es original de él, de hecho, es parte de la filosofía del siglo XX. La virtud de Quine es precisamente el hacer dicha crítica viendo que el empirismo conlleva dos dogmas por el hecho de ser incapaces de interpretar la reificación correctamente, en lugar de centrarse solo en la carga ontológica que conlleva en la metafísica. Los dos más importantes son las dos bases de las que parte su postura: la existencia de enunciados analíticos que escapen a la observación, y el reduccionismo que nos hace creer que los enunciados son traducibles a su contenido empírico directo. Son pues, 
el mismo error del conocimiento que ha sido recurrente en prácticamente todas las corrientes filosóficas y que, además, ha constituido una de las problemáticas más importantes del conocimiento.

\section{CONCLUSIÓN}

Como he analizado, Quine fundamenta su sistema del conocimiento en la reificación y la intersubjetividad. Sin embargo, no habría intersubjetividad sin capacidad de reificar, por lo que podríamos identificar la reificación como el origen del conocimiento, a pesar de no ser algo sucesivo. No estamos desprovistos de una alternativa a la metafísica o al empirismo lógico. De hecho, al haber demostrado que el empirismo sustenta sus bases en dos tesis metafísicas, Quine propone una nueva visión de un empirismo sin dogmas, basado siempre en la observación, pero liberado de los dos presupuestos que he analizado en el segundo apartado. Esta nueva visión del conocimiento es la que he recapitulado y sistematizado en el tercer apartado. En él se ven con fuerza las tesis pragmatistas que impregnan toda la teoría de Quine, así como sus críticas. Además, en este apartado he mostrado un giro epistémico respecto del holismo y la reificación. Si las interpretaciones del siglo XX consideran que el holismo es el eje central de la filosofía de Quine, aquí he mostrado que la reificación juega un papel igual o más importante que el holismo.

Quine propone un modo de ver el conocimiento como una red de relaciones provocada, en primer lugar, por múltiples entradas neuronales, que con la capacidad de reificación y la intersubjetividad de una misma comunidad de hablantes se convierten en enunciados observacionales. Estos enunciados observacionales pueden ligarse unos con otros para deducir categóricos observacionales. De este modo surgen las hipótesis, que serán puestas a prueba a través de los categóricos observacionales, así como de sus respectivos enunciados con el método hipotético-deductivo. Este es el método de avanzar en el conocimiento: es crucial tomar los enunciados de forma holística. La red de relaciones está sistematizada y jerarquizada, pero esta jerarquización no viene dada ni por su contenido empírico, ni por su valor de verdad metafísicos. Más bien, la jerarquización viene dada por su capacidad de revisión sin comprometer la totalidad de la estructura. El papel inicial de la reificación aquí es claro: proporcionar la capacidad de agrupar cualidades para poder formar enunciados observacionales o 
categóricos observacionales primitivos. Esta propuesta es un uso de la reificación mucho más cauteloso, en el que no caemos en el engaño de que lo que reificamos tiene existencia fuera de nuestro esquema conceptual.

En este trabajo, he realizado un extenso recorrido para llegar a la conclusión de que la reificación es el origen del error del que partimos a la hora de buscar y justificar el conocimiento. Es como se ha hecho desde los inicios de la historia del pensamiento precisamente porque es algo innato. Nos resulta muy difícil salirnos del esquema de la reificación y reparar en que es una particular capacidad de nuestra especie. Sin embargo, esto no tiene que llevar a confusión. La reificación no es algo negativo o peligroso que haya que evitar. Por el contrario, es, como se ha señalado, lo que posibilita el conocimiento en cada individuo. El problema no está en la reificación como tal, sino en la mala interpretación que la historia de la filosofía ha llevado a cabo de ella. Podemos añadir, para terminar, teniendo en cuenta las diferencias que Quine recoge y pone de manifiesto en toda su obra que, dentro de este esquema pragmatista del conocimiento, el lenguaje no crea el mundo, sino nuestra realidad, la cual no puede ser sino epistemológica. Conviene aquí recordar de nuevo lo que dije antes: la realidad nunca nos sobrepasa, sino que es una proyección nuestra.

María de la Esperanza Aguilar de la Morena es graduada en filosofía por la Universidad de Málaga.

\section{Lineas de investigación:}

Filosofía del siglo XX, Lacan, Filosofía Estructuralista.

Dirección electrónica: espe.aguilar.96@gmail.com 
